\title{
Results of the return air raise silencer system upgrade at Totten Mine
}

\author{
El Acuña Vale Canada Limited, Canada
}

A Dobson HGC Engineering, Canada

\begin{abstract}
The Totten Mine project specified two parallel main return air fans located on-surface equipped with baffle silencers designed to attenuate the sound levels from the fan outlets. In late 2014, an acoustical study determined that the return air fans were responsible for a series of noise disturbances in a community situated approximately $9 \mathrm{~km}$ to the northeast. The results of the acoustical study also indicated that the silencers were significantly underperforming due to clogging with a combination of particulate and moisture drawn from the underground mine. During 2015 and the beginning of 2016, Totten Mine implemented a cleaning procedure to mitigate the underperformance of the silencers. Unfortunately, noise monitoring of the cleaning procedures indicated diminishing results over time. As a result, a temporary solution was identified and implemented in Q3 and Q4 of 2016 to allow adequate time for engineering and defining of a long-term solution. The proposed temporary solution, however, had the potential to become the permanent solution, subject to an evaluation of performance. This paper presents the results of the temporary solution evaluation, when compared to the baseline cleaning procedure. The temporary solution is then compared to the proposed alternatives that were to be part of a feasibility study for both capital (CAPEX) and operational (OPEX) cost evaluation. The resulting long-term solution to manage noise issues on mine return air systems, similar to the Totten experience, is also presented and discussed.
\end{abstract}

Keywords: main return air raise fans, silencers, noise level measurements

\section{Introduction}

Totten Mine is located in the Sudbury Basin in the province of Ontario, Canada. The mine resumed operations at the end of 2013 and was equipped with two main return air fans and two main fresh air fans located on-surface. Both fresh air and return air fans are set in a parallel arrangement to work as integrated units and equipped with variable frequency drives (VFDs) to allow for the remote manual modification of their operating set point, usually between 600 to $880 \mathrm{rpm}$, depending on the mine's requirements. The main return air raise (RAR) fans are also equipped with silencers to attenuate the noise levels. The two most common set points, for both main return fans, are $800 \mathrm{rpm}$ during the summer months and $750 \mathrm{rpm}$ during the winter months. The main fresh air raise (FAR) fans' most common set points are $600 \mathrm{rpm}$ during winter and $650 \mathrm{rpm}$ during summer. In the event that one of the main fresh air fans needs a maintenance shut down, the remaining fresh air fan is set at $880 \mathrm{rpm}$ and the two main return air fans are set to $600 \mathrm{rpm}$ to compensate for the unavailable main fresh air fan. Similarly, if one of the two main return air fans needs to be shut down, the operational fan will be ramped up to $880 \mathrm{rpm}$ and the main fresh air fans set to $425 \mathrm{rpm}$, until both main return fans are operating together again.

At the beginning of 2015, acoustical monitoring detected that the noise level of the main return air fans was increasing as a result of the silencers becoming clogged due to moisture and particulates being drawn from underground. To mitigate this condition, a silencer cleaning process was put in place to remove the particulate material that was depositing on the internal surface of the silencers. The plan was intended to allow the mine site to develop and execute a phased engineered solution; it was not known how long the cleaning process would maintain its effectiveness, however, since the initial results were positive, sufficient time was available to scope various alternatives for a permanent solution. 
At the beginning of the second quarter of 2016, it became evident that the cleaning process was less effective and the silencers were exhibiting signs of associated deterioration. In response, a second intermediate solution was defined that had the potential to become a permanent solution subject to adequate performance results. The formulation of the intermediate solution involved the Totten engineering team, central ventilation group, and input from a local silencer manufacturer, SteelNorth.

The following sections summarise the noise reduction performance of the initial cleaning process implemented in 2015, the intermediate solution with its plan for execution, and the resulting noise measurements before and after the intermediate silencer was in place. A comparison of noise reduction, relative capital investment and maintenance costs is provided for the initial cleaning process and the intermediate solution. Based on the results obtained, the proposed decision to permanently accept the intermediate silencing system is discussed.

\section{$2 \quad$ Existing noise management in place and intermediate solution}

The increased noise level detected in 2015 required Totten Mine to find a quick, effective and efficient solution. The first step was to contact the manufacturer and supplier of the return air fan system to provide solutions for consideration. The initial solution was to undertake a silencer cleaning process to prolong the effective life of the current silencing infrastructure on a temporary basis, until a new solution could be determined. The cleaning was able to be done immediately but was not efficient in terms of the use of resources over time. As soon as the cleaning process was in place, Totten Mine started investigating longer term alternatives. By the time the deterioration of the silencer and the diminishing results of the cleaning process were evident, Totten Mine had identified several potential solutions. The following section describes the initial cleaning and the new solution developed with the local silencer manufacturer previously mentioned.

\subsection{Silencer cleaning process}

The cleaning process consisted of dismounting the clogged silencer from its piers onto a separate supporting structure and pressure washing the internal silencer surface with a mix of water and soap, then relocating the silencer to its original place. The cleaning process was performed individually for each main return air fan silencer and it required the main return air fan to be shut down. One day shift of work was required per silencer for the cleaning process, resulting in two days to complete. In addition to time, the process required the support of multiple outside resources such as personnel for construction and cleaning, vacuum cleaners for the waste water that was transported to the treatment plant, and a crane. Overall, three contractors working in co-ordination, plus mine supervision, were required. The cleaning process was costly, but relatively quick, and was initially required twice a year.

Table 1 presents the noise level result of three cleaning processes that were performed between April 2015 and April 2016 (Dobson \& Acuña 2017) for the return air silencers. The last column in Table 1 (dBA) aligns each cleaning with a respective noise reduction; 9,8 and $6 \mathrm{dBA}$. Measurements were conducted in five positions $\left(0,22.5,45,67.5\right.$, and $\left.90^{\circ}\right)$ at $50 \mathrm{~m}$ from the outlet. Measurement positions were marked and identical, for each dataset presented.

The results in Table 1 suggest that consecutive cleaning processes and continued exposure to the moist underground airflow were deteriorating the silencers and affecting the performance. Without other alternatives, this would mean increasing the frequency of silencer cleaning. Increasing silencer cleaning frequency was not desirable from the perspective of silencer deterioration, so an alternate operating plan was required before noise levels exceeded allowable limits.

Based on the experience gained with the original silencers, two main criteria for the design of new replacement silencers were defined. These criteria included the requirement for interchangeable baffles that could be dismounted without removing the full silencer structure and the ability for baffle cleaning or replacement according to requirements. 
Table 1 Measured sound power levels of Totten Mine RAR outlets ( $\mathrm{dB}$ re $10^{-12}$ watt) before and after cleaning the silencers, fan speed of 750 rpm (Dobson \& Acuña 2017)

\begin{tabular}{|c|c|c|c|c|c|c|c|c|c|}
\hline \multirow{2}{*}{ Measurement date and silencer condition } & \multicolumn{8}{|c|}{ Octave band centre frequency $(\mathrm{Hz})$} & \multirow{2}{*}{ dBA } \\
\hline & 63 & 125 & 250 & 500 & $1 \mathbf{k}$ & $2 k$ & 4k & 8k & \\
\hline 4 March 2015 - before cleaning (dB) & 131 & 125 & 125 & 127 & 122 & 115 & 106 & 96 & 128 \\
\hline 7 April 2015 - after cleaning (dB) & 110 & 109 & 117 & 117 & 114 & 105 & 97 & 88 & 119 \\
\hline Sound level reduction $(\mathrm{dB})$ & 21 & 16 & 8 & 10 & 8 & 10 & 9 & 8 & 9 \\
\hline 3 November 2015 - before cleaning (dB) & 112 & 112 & 123 & 126 & 118 & 109 & 101 & 95 & 124 \\
\hline 6 November 2015 - after cleaning (dB) & 112 & 107 & 113 & 116 & 111 & 104 & 98 & 92 & 116 \\
\hline Sound level reduction $(\mathrm{dB})$ & - & 5 & 10 & 10 & 7 & 5 & 3 & 3 & 8 \\
\hline 11 April 2016 - before cleaning (dB) & 110 & 111 & 123 & 126 & 117 & 108 & 102 & 94 & 124 \\
\hline 14 April 2016 - after cleaning (dB) & 110 & 111 & 117 & 118 & 114 & 104 & 98 & 89 & 118 \\
\hline Sound level reduction $(\mathrm{dB})$ & - & - & 6 & 8 & 3 & 4 & 4 & 5 & 6 \\
\hline
\end{tabular}

\subsection{Design of new silencer and implementation plan}

During the time that the initial silencers were undergoing the periodic cleaning process, alternative options were studied to understand costs, construction requirements and schedules. The implementation timelines for a project of this size considering engineering, procurement, construction and installation could range between one and two years. As a result, another intermediate solution was necessary that could be put in place until a permanent system was developed and installed.

SteelNorth currently supplies mine sites with silencers for underground axial auxiliary fans; their noise reduction performance had been confirmed by Vale occupational health personnel to significantly reduce noise levels at a competitive cost. The silencers installed on underground auxiliary fans ranged in size from $0.45 \mathrm{~m} \mathrm{(18")}$ to $1.37 \mathrm{~m} \mathrm{(54")}$ in diameter. Although the Totten Mine primary fans are also axial, they are

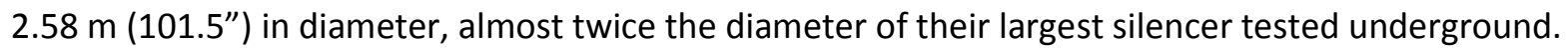

Based on their history and previous experience in building silencers for auxiliary and main fans, Totten Mine approached the company with design criteria to meet an intermediate solution. The manufacturer was willing to provide a silencer with a guarantee of performance.

The intermediate silencer design was required to meet the following:

- Noise level reduction from the new silencer, once installed, must produce performance results equal to or lower than the measured noise levels of the previous cleaning processes as presented in Table 1.

- The pressure drop across the silencer must be equal to or lower than the pressure loss across the original silencing system. Totten Mine has a pressure sensor installed on-surface at the return air raise that was used for this purpose.

- The durability of the new solution must be equal to or better than the original silencing system, with regards to cleaning time, procedure and cost.

Based on the performance of the intermediate silencer, the design could potentially become a permanent solution. In order for this to occur, additional conditions would need to be satisfied, for example:

- The proposed solution must be within CAPEX and OPEX budgetary constraints compared to the original cleaning process and the replacement alternatives identified by Totten Mine that were considered to have a relatively high budgetary estimate. 
- Meet previous conditions and show a net present value (NPV) favourable to the current cleaning process.

- The intermediate silencing system would show an improvement compared to the current cleaning process, both in terms of time and resources. This is necessary to reduce delays to production caused by the cleaning process.

To minimise the risk of implementing a new design, the silencer was installed on one return fan first and then it was tested for performance before the second silencer was built and installed on the second parallel fan.

An execution plan, budget and schedule was defined to deliver and install the silencers between May and November 2016, which included an interdisciplinary effort from mine management, project management, surface fixed facility, warehouse, central engineering, mine ventilation, central ventilation, purchasing, local supplier, contractors and others.

\section{Silencer performance and achieved savings (CAPEX and OPEX)}

The silencer manufacturer was able to provide a solution that met the specifications, budget and schedule established by Totten Mine. The following sections describe the solution proposed, measured noise levels to date (March 2017) compared to the historical data, and economic analysis of the new solution compared to the cleaning process.

\subsection{New silencer solution delivered}

Considering the mine requirements, the manufacturer proposed and developed a design with the following characteristics:

- Multiple piece structure, which allowed transportation without the need for special oversized load traffic permits.

- Incorporation of a two-silencer cassette design which also allowed installation without the use of a crane. The forklift at the mine site was able to quickly mount and dismount the unit.

- Removable silencing baffles for easy replacement and offsite cleaning. Only the two silencing cassettes need to be dismounted, sent for cleaning or rebuild and then returned to be ready for the next cleaning cycle.

- Elimination of the onsite cleaning process which could be completed offsite in a more suitable environment.

These key points of the new design created an improvement in terms of time and resources as the silencer change could be performed in a couple of hours.

\subsection{Noise reduction comparisons}

Noise level measurements were performed by HGC Engineering before and after the first new silencer was installed on the south RAR outlet. The results presented in Table 2 consider one new silencer installed beside one original silencer on the parallel fan arrangement, however, only the south main fan with the new silencer was operating during the measurements. 
Table 2 Measured sound power levels of Totten Mine RAR south outlet (dB re $10^{-12}$ watt) before and after installation of the new silencer, fan speed of 750 and $880 \mathrm{rpm}$

\begin{tabular}{lllllllllll}
\hline & \multicolumn{1}{c}{ Octave band centre frequency (Hz) } & & dBA \\
\cline { 2 - 9 } Measurement date and silencer condition & $\mathbf{6 3}$ & $\mathbf{1 2 5}$ & $\mathbf{2 5 0}$ & $\mathbf{5 0 0}$ & $\mathbf{1 k}$ & $\mathbf{2 k}$ & $\mathbf{4 k}$ & $\mathbf{8 k}$ & \\
\hline Old silencer (28 September 2016) 750 rpm & 104 & 108 & 108 & 114 & 110 & 102 & 95 & 93 & 120 \\
New silencer (28 September 2016) 750 rpm & 110 & 108 & 102 & 102 & 95 & 92 & 89 & 88 & 109 \\
Sound level reduction (dB) 750 rpm & $\mathbf{- 6}$ & $\mathbf{0}$ & $\mathbf{6}$ & $\mathbf{1 2}$ & $\mathbf{1 5}$ & $\mathbf{1 0}$ & $\mathbf{6}$ & $\mathbf{5}$ & $\mathbf{1 1}$ \\
Old silencer (28 September 2016) 880 rpm & 107 & 111 & 125 & 123 & 114 & 107 & 100 & 98 & 125 \\
New silencer (28 September 2016) 880 rpm & 112 & 107 & 113 & 106 & 96 & 93 & 89 & 89 & 110 \\
Sound level reduction (dB) 880 rpm & $\mathbf{- 5}$ & $\mathbf{3}$ & $\mathbf{1 2}$ & $\mathbf{1 7}$ & $\mathbf{1 9}$ & $\mathbf{1 4}$ & $\mathbf{1 1}$ & $\mathbf{9}$ & $\mathbf{1 6}$ \\
\hline
\end{tabular}

As presented in Table 2 the noise reduction of the new silencer on the south outlet was measured at 750 and $880 \mathrm{rpm}$. The new silencer resulted in a source noise level at or under $110 \mathrm{dBA}$ in both operational speed conditions, and was therefore outperforming the original clogged silencer. With both fans performing at the same rpm, air volume and temperature, the pressure measurement at the collar before the silencer change was $1.2 \mathrm{kPa}$ (4.8 in. wg (inches of water gauge)) and after the change it was $1.05 \mathrm{kPa}$ (4.2 in. wg). Considering the positive results of the first silencer replacement test, it was decided to proceed with the second replacement silencer on the north RAR outlet. At the time of the writing, the durability and sustained performance of the new silencer had not been assessed. Table 3 compares the measured noise levels of the RAR with the original silencers and the new silencers on both outlets, after the second silencer was installed on the north outlet in November of 2016.

Table 3 Measured sound power levels of both Totten Mine RAR outlets compared ( $\mathrm{dB}$ re $10^{-12}$ watt) old silencers (after cleaning) with new silencers (fan speed of 750 and $880 \mathrm{rpm}$ )

\begin{tabular}{|c|c|c|c|c|c|c|c|c|c|}
\hline \multirow{2}{*}{ Measurement date and silencer condition } & \multicolumn{8}{|c|}{ Octave band centre frequency $(\mathrm{Hz})$} & \multirow{2}{*}{ dBA } \\
\hline & 63 & 125 & 250 & 500 & $1 \mathrm{k}$ & $2 \mathbf{2 k}$ & $4 k$ & 8k & \\
\hline Old silencers after cleaning ( 7 April 20 & & 109 & 117 & 117 & 114 & 105 & 97 & 88 & 119 \\
\hline Two new silencers (16 Nov. 2016) 750 & 114 & 111 & 115 & 115 & 108 & 101 & 96 & 91 & 115 \\
\hline Sound level reduction (dB) $750 \mathrm{rpm}$ & -4 & -2 & 2 & 2 & 6 & 4 & 1 & -3 & 4 \\
\hline Old silencers after cleaning ( 6 Nov. 2015) 750 & 112 & 107 & 113 & 116 & 111 & 104 & 98 & 92 & 116 \\
\hline Two new silencers (16 Nov. 2016) 750 rpm & 114 & 111 & 115 & 115 & 108 & 101 & 96 & 91 & 115 \\
\hline Sound level reduction (dB) $750 \mathrm{rpm}$ & -2 & -4 & -2 & 1 & 3 & 3 & 2 & 1 & 1 \\
\hline Old silencers after cleaning (14 April 2016) 75 & 110 & 111 & 117 & 118 & 114 & 104 & 98 & 89 & 118 \\
\hline 6) $750 \mathrm{rpm}$ & 1 & 111 & 115 & 110 & 108 & 101 & 96 & 91 & 115 \\
\hline Sound level reduction (dB) $750 \mathrm{rpm}$ & -4 & 0 & 2 & 3 & 6 & 3 & 2 & -2 & 3 \\
\hline Old silencers after cleaning (14 April 2016) 880 rpm & 116 & 116 & 123 & 121 & 119 & 110 & 104 & 94 & 123 \\
\hline Two new silencers (16 November 2016) 880 rpm & 117 & 113 & 118 & 119 & 113 & 107 & 100 & 95 & 119 \\
\hline Sound level reduction $(\mathrm{dB}) 880 \mathrm{rpm}$ & -1 & 3 & 5 & 2 & 6 & 3 & 4 & -1 & 4 \\
\hline
\end{tabular}

Table 3 presents four sets of measurements, under similar conditions, of the new silencers compared to the performance of the original silencers after cleaning. As can be observed, the new silencers 
outperformed the original system after the cleaning process. This was sufficient to meet condition one of the noise design criteria.

With both fans performing at the same rpm, air volume and temperature, the pressure measurement before the silencer change was $0.75 \mathrm{kPa}$ (3.0 in. wg) and after the change it was $0.75 \mathrm{kPa}(3.0 \mathrm{in} . \mathrm{wg})$. Although the differential pressure measurement did not show less resistance from the two new silencers in the main return air fan system, it was considered sufficient to meet condition two of the pressure drop criteria.

Again, durability and sustained performance will need to be assessed over the upcoming months to determine an appropriate cleaning cycle, and to determine whether the subject noise control system will meet all requirements to become the permanent solution.

During the second round of noise measurements (November 2016), the silencer replaced initially on the south RAR outlet (in September 2016) was measured individually to assess deterioration in noise attenuation performance due to clogging. Deterioration could be observed, however, it was within the range of what had been previously noticed through the cleaning processes of the original silencers. It could also be observed that particulate material and moisture from underground was adhering to the walls of the silencer baffles.

\subsection{Economic results (CAPEX and OPEX)}

Both new silencers were installed according to the timelines of the initial schedule. Based on the high-level budget estimates received by Totten Mine for an alternative solution with the associated engineering and construction costs (CAPEX), the design proposed by the local silencer manufacturer generated savings in the range of $80 \%$ compared to an equivalent solution.

Based on the cost of cleaning the original silencers and the current proposed cost to rebuild the silencer cassettes, the operational cost (OPEX) was reduced by at least $35 \%$ with the consideration of only two cleaning processes per year.

The internal cost incurred by the mine to perform the cleaning process of the new silencer was not estimated, but it was evident to the personnel involved in the project that the new process in place will require less personnel and resources to accomplish the same end result. Based on this, it was considered that all conditions imposed by Vale's Totten Mine were met.

\section{Conclusion}

A new silencer solution has been implemented at Totten Mine for the main return air fans to mitigate the noise level concerns associated with the clogging of the original silencers with particulate material and moisture coming from underground. This new silencer design has considerable benefits relative to the previous solution of cleaning the original silencers, and has the ability to perform at equal or lower noise levels. Therefore, the new design has the potential to be implemented as a permanent solution, which will be confirmed in the upcoming months through the acquisition of measured data.

The proposed solution has demonstrated to also have a significantly lower CAPEX and OPEX cost compared to the previous cleaning process. The new proposed solution appears to be more robust than the previous one in terms of cost, implementation time and required mine resources. One lesson learned from this successful implementation is that a reasonable solution can be developed with a local supplier that has developed and proven technical experience. Sudbury is fortunate to have a significant number of such suppliers with relevant background experience and the desire to take on the associated technical challenges to deliver a new solution with evolving requirements and budget constraints.

\section{References}

Dobson, A \& Acuña, El 2017, 'Planning the upgrade of the return air raise silencer system at Totten Mine', in JF Brune (ed.), Proceedings of the 16th North American Mine Ventilation Symposium, Colorado School of Mines, Golden, chapter 16, pp. 9-16. 